\title{
Complexity of Presburger Arithmetic with Fixed Quantifier Dimension
}

\author{
Uwe Schöning \\ Universität Ulm \\ D-89069 Ulm
}

Abt. Theoretische Informatik

\begin{abstract}
It is shown that the decision problem for formulas in Presburger arithmetic with quantifier prefix $\left[\exists_{1} \forall_{2} \ldots \exists_{m} \forall^{3}\right]$ (for $m$ odd) and $\left[\exists_{1} \forall_{2} \ldots \forall_{m} \exists^{3}\right]$ (for $m$ even) is complete for the class $\Sigma_{m}^{P}$ of the polynomial-time hierarchy. Furthermore, the prefix type $[\exists \forall \exists \exists]$ is complete for $\Sigma_{2}^{P}$, and the prefix type $[\exists \forall]$ is complete for NP. This improves results (and solves a problem left open) by Grädel [7].
\end{abstract}

\section{Introduction}

We assume familiarity with the standard classes in complexity theory, like P and NP, the classes $\Sigma_{m}^{P}$ of polynomial-time hierarchy, and the notion of polynomial-time reduction (see $[6,9])$.

Let 3-CNF (3-DNF) denote the set of propositional formulas in conjunctive (disjunctive) normal form where each clause consists of 3 literals. A literal is a Boolean variable or a negated Boolean variable.

The decision problem $B_{m}$ consists of Boolean formulas of the form $F\left(X^{1}, \ldots, X^{m}\right)$ where each $X^{i}$ is a separate sequence of Boolean variables, $X^{i}=\left(x_{1}^{i}, \ldots, x_{n_{i}}^{i}\right)$, such that

$$
\exists X^{1} \forall X^{2} \ldots Q_{m} X^{m} F\left(X^{1}, X^{2}, \ldots, X^{m}\right)
$$

is true. It is known that for each $m \geq 1, B_{m}$ is complete for $\Sigma_{m}^{P}$ (cf. $\left.[12,13]\right)$.

Presburger arithmetic is the first-order theory of the natural numbers with addition. Let $P A$ denote the set of formulas which are true in this interpretation. It is known that $P A$ has double-exponential complexity on alternating Turing machines (cf. $[4,3,1]$ ) whereas the complexity of $P A_{m}$, the set of true Presburger formulas with $m$ quantifier alternations, is roughly one exponential step lower than the general case (cf. $[10,5,7])$.

Formulas with fixed dimension are obtained by fixing the quantifier prefix (and therefore also the number of alternations) to a certain type which we will denote by 
$\left[Q_{1} Q_{2} \ldots Q_{m}\right], Q_{i} \in\{\exists, \forall\}$. Grädel has obtained in his dissertation [7] several $\Sigma_{m}^{P}$ completeness results for decision problems of the form $\left[Q_{1} Q_{2} \ldots Q_{k}\right] \cap P A, k>m$. More precisely, he shows that

$$
\begin{aligned}
& {\left[\exists_{1} \forall_{2} \ldots \exists_{m} \exists^{2} \forall^{3}\right] \cap P A \text { is complete for } \Sigma_{m}^{P} \text { (if } m \text { is odd), }} \\
& {\left[\exists_{1} \forall_{2} \ldots \forall_{m} \forall^{2} \exists^{3}\right] \cap P A \text { is complete for } \Sigma_{m}^{P} \text { (if } m \text { is even). }}
\end{aligned}
$$

For the case $m=1$ he can obtain a stronger result:

$[\exists \forall \forall] \cap P A$ is complete for NP.

By swapping universal and existential quantifiers one can, of course, obtain a dual $\Pi_{m}^{P}$ completeness result. Furthermore, the same complexity status as listed above have all such prefix classes which extend the above ones by finitely many quantifiers and do not increase the number of alternations. Therefore, the complexity status of all but finitely many prefix types is resolved in terms of a completeness result in the polynomial-time hierarchy. Some prefix types remain open, especially Grädel poses the open problem what the status of $[\exists \forall] \cap P A$ is.

In this paper we will stengthen the above completeness results by including more prefix types, and we resolve thereby the complexity status of $[\exists \forall] \cap P A$; it is NPcomplete.

\section{Main Result}

Theorem. For each $m \geq 1$, the language $\left[\exists \forall \ldots \exists_{m} \forall^{3}\right] \cap P A$ (for $m$ odd) and $\left[\exists \forall \ldots \forall_{m} \exists^{3}\right] \cap P A$ (for $m$ even) is $\Sigma_{m}^{P}$-complete.

Proof: Membership in $\Sigma_{m}^{P}$ is shown in [7] (relying on results in [8, 11, 10]).

For the following we assume that $m$ is odd. In this case the problem $B_{m} \cap 3-\mathrm{CNF}$ is complete for $\Sigma_{m}^{P}$ (see $\left.[12,13]\right)$. (For the case of $m$ even, we need to consider the problem $B_{m} \cap 3$-DNF instead. The proof in this case is virtually the same.)

Let $F=F\left(X^{1}, X^{2}, \ldots, X^{m}\right)$ be a formula in 3-CNF where the $X^{i}$ are sequences of Boolean variables, $X^{i}=\left(x_{1}^{i}, \ldots, x_{k}^{i}\right)$. We assume without loss of generality that each variable sequence $X^{i}$ consists of the same number of variables, namely $k$.

Let

$$
p_{1}, p_{2}, \ldots, p_{k}
$$

be the sequence of the first $k$ primes. It is important to notice that this sequence can be constructed in polynomial-time, relative to the size of $F$.

For the intended reduction, we want to map the formula

$$
\exists X^{1} \forall X^{2} \ldots \exists X^{m} F\left(X^{1}, X^{2}, \ldots, X^{m}\right)
$$


to a formula in Presburger arithmetic of the following form

$$
\exists z_{1} \forall z_{2} \ldots \exists z_{m} G\left(z_{1}, z_{2}, \ldots, z_{m}\right)
$$

where the $z_{i}$ are variables that represent natural numbers (and encode the assignments $X^{i}$ ) and $G$ is a Presburger formula intended to check whether these assignments make $F$ true. A Boolean assignment $\left(x_{1}, \ldots, x_{k}\right) \in\{0,1\}^{k}$ will be represented by a number $z$ that satisfies the set of modular equations

$$
\begin{gathered}
z \equiv x_{1}\left(\bmod p_{1}\right) \\
z \equiv x_{2}\left(\bmod p_{2}\right) \\
\vdots \\
z \equiv x_{k}\left(\bmod p_{k}\right)
\end{gathered}
$$

The existence of such a $z<\prod_{j=1}^{k} p_{j}$ is guaranteed by the Chinese remainder theorem.

We need to construct a Presburger formula $A(z)$ that evaluates to true if and only if the number $z$ correctly represents a Boolean assignment, in the sense above. We need to express that for $j=1, \ldots, k$ it holds that $\left(z \bmod p_{j}\right) \in\{0,1\}$. Therefore $A(z)$ has the following, tentative form

$$
\bigwedge_{j=1}^{k}\left[\left(z \bmod p_{j}\right) \in\{0,1\}\right]
$$

Equivalently,

$$
\bigwedge_{j=1}^{k} \bigwedge_{k=2}^{p_{j}-1}\left[z \not \equiv k\left(\bmod p_{j}\right)\right]
$$

The expression in brackets can be rewritten as a formula in Presburger arithmetic:

$$
\forall u\left(p_{j} \cdot u+k \neq z\right)
$$

where the notation $p_{j} \cdot u$ is an abbreviation for $u+u+\cdots+u$ ( $p_{j}$ times). The universal quantifier can be pulled in front, so that the formula for $A(z)$ gets the final form

$$
\forall u\left[\bigwedge_{j=1}^{k} \bigwedge_{k=2}^{p_{j}-1}\left(p_{j} \cdot u+k \neq z\right)\right]
$$

The intended formula

$$
\exists z_{1} \forall z_{2} \ldots Q_{m} z_{m} G\left(z_{1}, z_{2}, \ldots, z_{m}\right)
$$

is indeed equivalent to the following form

$$
\begin{array}{ccccc}
\exists z_{1} & \left(A\left(z_{1}\right)\right. & \wedge & & \\
\\
\forall z_{2} & \left(A\left(z_{2}\right)\right. & \rightarrow & & \\
& \exists z_{3} & \left(A\left(z_{3}\right)\right. & \wedge & \\
& & & \ddots & \\
& & & & \left.\left.H\left(z_{1}, z_{2}, \ldots, z_{m}\right) \ldots\right)\right)
\end{array}
$$


We have seen that $A\left(z_{i}\right)$ can be expressed by one universal quantifier. This enables us in this case to merge quantifiers of the same type into one quantifier. In particular, the universal quantifier in $A\left(z_{1}\right)$ can be melted together with " $\forall z_{2}$ " since they are connected conjunctively. Similarly, the existential quantifier that we need to express " $A\left(z_{2}\right) \rightarrow \ldots$. can be melted together with " $\exists z_{3}$ ", and so on. Altogether, we get a quantifier prefix (before the beginning of the formula $H$ ) of the form $\left[\exists_{1} \forall_{2} \ldots \exists_{m} \forall\right]$.

The Presburger formula $H$ is intended to express the fact that $F$ is satisfied by the assignments $\left(X^{1}, \ldots, X^{m}\right)$. This formula consists of a conjunction of formulas,

$$
H=\bigwedge_{i=1}^{n} C_{i}
$$

where $C_{i}$ expresses in Presburger arithmetic that the $i$ th clause in $F$ is satisfied. As a concrete example, let the literals of this clause be $x_{3}^{1}, \neg x_{1}^{2}, x_{2}^{2}$. We can then, tentatively, express $C_{i}$ as

$$
\neg\left[\left(z_{1} \equiv 0\left(\bmod p_{3}\right)\right) \wedge\left(z_{2} \equiv 1\left(\bmod p_{1}\right)\right) \wedge\left(z_{2} \equiv 0\left(\bmod p_{2}\right)\right)\right]
$$

Furthermore, we can combine subformulas which start with the same $z_{i}$. By the Chinese remainder theorem, there is a number $a<p_{1} \cdot p_{2}$ (which can be efficiently computed, see [2] page 824) such that the above formula is equivalent to

$$
\neg\left[\left(z_{1} \equiv 0\left(\bmod p_{1}\right)\right) \wedge\left(z_{2} \equiv a\left(\bmod p_{1} \cdot p_{2}\right)\right)\right]
$$

This can be expressed in Presburger arithmetic as

$$
\begin{aligned}
& \neg\left[\exists u\left(p_{1} \cdot u=z_{1}\right) \wedge \exists v\left(p_{1} p_{2} \cdot v+a=z_{2}\right)\right] \\
\equiv & \forall u \forall v\left[\left(p_{1} \cdot u \neq z_{1}\right) \vee\left(p_{1} p_{2} \cdot v+a \neq z_{2}\right)\right]
\end{aligned}
$$

Like in this specific example we can, in general, express $C_{i}$ by a formula with one, two or three universal quantifiers, depending on the number of different $X^{i}$ 's (respectively $z_{i}^{\prime}$ 's) in that clause.

These up the 3 universal quantifiers per clause can be moved in front of the whole conjunction such that $H$ gets the following form

$$
\forall u \forall v \forall w\left[\bigwedge_{i=1}^{n}(\ldots)\right]
$$

Now the whole resulting formula has the following structure:

$$
\exists z_{1} \forall z_{2} \ldots \exists z_{m}\left(A\left(z_{m}\right) \wedge \forall u \forall v \forall w[\ldots]\right)
$$

Again we can melt together two quantifiers, namely the universal quantifier in $A\left(z_{m}\right)$ and " $\forall u$ ". So the final form of the Presburger formula has the quantifier prefix $\left[\exists_{1} \forall_{2} \ldots \exists_{m} \forall^{3}\right]$. Finally, we remark that the reduction can be carried out in polynomial time.

Inspecting the proof, by the fact that the number of different $X^{i}$ 's determines the last block of universal quantifiers, we get the following corollary. 
Corollary. The decision problem $[\exists \forall \exists \exists] \cap P A$ is $\Sigma_{2}^{P}$-complete.

The decision problem $[\exists \forall] \cap P A$ is NP-complete.

Remark: The NP-completeness of $[\exists \forall] \cap P A$ could also be obtained by a reduction from some other NP-complete problem; for this, Grädel (personal communication) has proposed problem [AN2] from [6].

\section{References}

[1] L. Berman. The complexity of logical theories. Theoretical Computer Science 11 (1980) $71-77$.

[2] T.H. Cormen, C.E. Leiserson, R.L. Rivest. Introduction to Algorithms. MIT Press, 1990.

[3] J. Ferrante, C.W. Rackoff. The Computational Complexity of Logical Theories. Lecture Notes in Mathematics 718, Springer, 1979.

[4] M.J. Fischer, M.O. Rabin. Super-exponential complexity of Presburger arithmetic. SIAM-AMS Proceedings 7 (1974) 27-41.

[5] M. Fürer. The complexity of Presburger arithmetic with bounded quantifier alternation depth. Theoretical Computer Science 18 (1982) 105-111.

[6] M.R. Garey, D.S. Johnson. Computers and Intractability: A Guide to the Theory of NP-Completeness. Freeman, 1979.

[7] E. Grädel. The Complexity of Subclasses of Logical Theories. Dissertation, Universität Basel, 1987.

[8] H. Lenstra. Integer programming with a fixed number of variables. Math. of Operations Research 8 (1983) 538-548.

[9] C. Papadimitriou. Computational Complexity. Addison-Wesley, 1994.

[10] C.R. Reddy, D.W. Loveland. Presburger arithmetic with bounded quantifier alternation. Proc. of the 10th ACM Symposium on Theory of Computing 1978, 320-325.

[11] B. Scarpellini. Complexity of subcases of Presburger arithmetic. Transactions of the AMS 284 (1984) 203-218.

[12] L. Stockmeyer. The polynomial-time hierarchy. Theoretical Computer Science 3 (1977) 1-22.

[13] C. Wrathall. Complete sets and the polynomial-time hierarchy. Theoretical Computer Science 3 (1977) 23-33. 